\title{
On the Interpretation and Correlation of High-Temperature Ignition Delays in Reactors with Varying Thermodynamic Conditions
}

\author{
MINGYUAN TAO, ${ }^{1}$ ANDREW LAICH, ${ }^{2}$ PATRICK LYNCH, ${ }^{2}$ PENG ZHAO ${ }^{1}$ \\ ${ }^{1}$ Department of Mechanical Engineering, Oakland University, Rochester, MI, 48309 \\ ${ }^{2}$ Department of Mechanical Engineering, University of Michigan-Dearborn, Dearborn, MI, 48128
}

Received 15 May 2017; revised 27 February 2018; accepted 1 March 2018

DOI 10.1002/kin.21170

Published online 6 April 2018 in Wiley Online Library (wileyonlinelibrary.com).

\begin{abstract}
Ignition delay time (IDT) is a useful global metric for fuel performance screening and a major target for kinetic modeling. Measurements of IDT are conceptually straightforward; however, interpretation could be complicated, especially for systems with changing temperature and pressure. Some experimental conditions in the high repetition rate miniature shock tube (HRRST) exhibit complex temperature and pressure state histories. To better interpret and correlate IDTs, especially those obtained in reactors with varying thermodynamic conditions, an inverse Livengood-Wu (L-W) integral technique is applied to deconvolve the constant condition IDTs from measured IDTs in the HRRST using information on the varying state history. In this paper, the approximate problem is demonstrated using only the measured pressure history
\end{abstract}

Correspondence to: Patrick Lynch; e-mail: ptlynch@uic.edu, Peng Zhao; e-mail: pengzhao@oakland.edu.

Present address of Patrick Lynch: Department of Mechanical and Industrial Engineering, University of Illinois at Chicago, Chicago, IL 60607, USA

Supporting Information is available in the online issue at www.wileyonlinelibrary.com.

(C) 2018 Wiley Periodicals, Inc.
Disclaimer: "This report was prepared as an account of work sponsored by an agency of the United States Government. Neither the United States Government nor any agency thereof, nor any of their employees, makes any warranty, express or implied, or assumes any legal liability or responsibility for the accuracy, completeness, or usefulness of any information, apparatus, product, or process disclosed, or represents that its use would not infringe privately owned rights. Reference herein to any specific commercial product, process, or service by trade name, trademark, manufacturer, or otherwise does not necessarily constitute or imply its endorsement, recommendation, or favoring by the United States Government or any agency thereof. The views and opinions of authors expressed herein do not necessarily state or reflect those of the United States Government or any agency thereof." 
leading up to ignition, where the temperature history is estimated based on an isentropic assumption. The IDTs of several fuels were first measured in the HRRST including isooctane and acetone to represent those fuels without strong low-temperature chemistry effects. Based on the measured pressure and approximated temperature history, measurements of IDTs in the HRRST compare very favorably with those measured using more conventional techniques, including conventionally sized shock tubes, via the inverse L-W correlation with relaxed Newton iteration and genetic algorithm. This study demonstrates the feasibility of using a high throughput ignition testing facility, like the HRRST to extract the constant state IDT measured in regular shock tubes, assisted by the inverse L-W correlation. It is expected with an independent temperature measurement available in the future, IDTs in a broader range of thermodynamic conditions and effects of heat loss could be better resolved. (c) 2018 Wiley Periodicals, Inc. Int J Chem Kinet 50: 410-424, 2018

\section{INTRODUCTION}

One of the most critical fuel properties and targets for the development and validation of a chemical mechanism is ignition delay time (IDT) at different conditions. Nearly-homogeneous facilities including shock tubes [1], rapid compression machines (RCM) [2], flow reactors [3], and homogeneous charge compression ignition (HCCI) engines [4,5] have been used to provide ignition performance testing under different thermodynamic conditions and flow residence time. The shock tube in particular is excellent for measuring ignition delay and resolving chemistry because of well understood fluid mechanics properties and tight control and selectability of thermodynamic conditions. In principle, the recently developed miniature high repetition rate shock tube (HRRST) [6] could be a useful extension as a high throughput testing facility for measuring IDT. The HRRST would be limited to hightemperature conditions due to the short test time, but other effects complicate interpretation of the data and comparison to IDTs measured in other facilities, including conventionally sized shock tubes. These complications include exacerbated boundary layer and gas dynamic effects, etc. leading to changing temperature and pressure history before ignition. Developing the formalism for interpretation and cross-validation of HRRST IDT data is one focus of this work, but the problem of interpretation and cross-validation of data among different reactors with different varying thermodynamic conditions is a broader problem. This interpretation method can be used to reduce the so called "facility effects."

An early attempt at extracting kinetic detail, by considering IDT $\tau(T, P)$ as a measure of the chemical reactivity of a homogeneous mixture under constant volume conditions characterized by initial $P$ and $T$, Livengood and $\mathrm{Wu}$ [7] proposed a predictive integral method based on the accumulation of a chain carrier to correlate the autoignition timing, for example, $\tau^{\prime}$ in IC engines, with those in RCMs by integrating over the thermodynamic path using the formula:

$$
1=\int_{0}^{\tau^{\prime}} \frac{d t}{\tau(T(t), P(t))}
$$

This method, hereafter referred to as L-W correlation, has been widely used in the prediction and correlation of autoignition in IC engines due to its simplicity and accuracy, especially where the ignition delay can be well described by single-step Arrhenius-like kinetics under high-temperature conditions. It should be noted that for high-T applications, the preignition heat release is usually very small such that there is no obvious difference in the constant volume and constant pressure ignition delay, thus it is frequently referred as constant state IDT. That is what is being identified in the current study. On the other hand, it is also well known that during autoignition of fuel-oxidizer systems, the ignition response can vary nonmonotonically with the global parameters $(P, T)$ due to the variation of controlling chemistry, such as the negative temperature coefficient (NTC) response for general large hydrocarbon fuels. While the base L-W method is inappropriate for describing these effects, it is worth noting that, to address the NTC-affected ignition, low-temperature chemistry can be further included. Cool flame reactivity and two-stage ignition under advanced compression ignition conditions can be accurately predicted using a staged L-W integral method [8,9]. Empirical correlation of IDT under constant volume has been obtained based on conventional L-W [10] including NTC, which is further adopted in a recent work [11] to correlate the IDT of fuel blends based on individual components and mixture composition. It should be noted that the low-temperature and blending extension is the focus of ongoing work, and the current work is limited to high-temperature regions. Also, although substantial progress has been made by kinetic model developers 


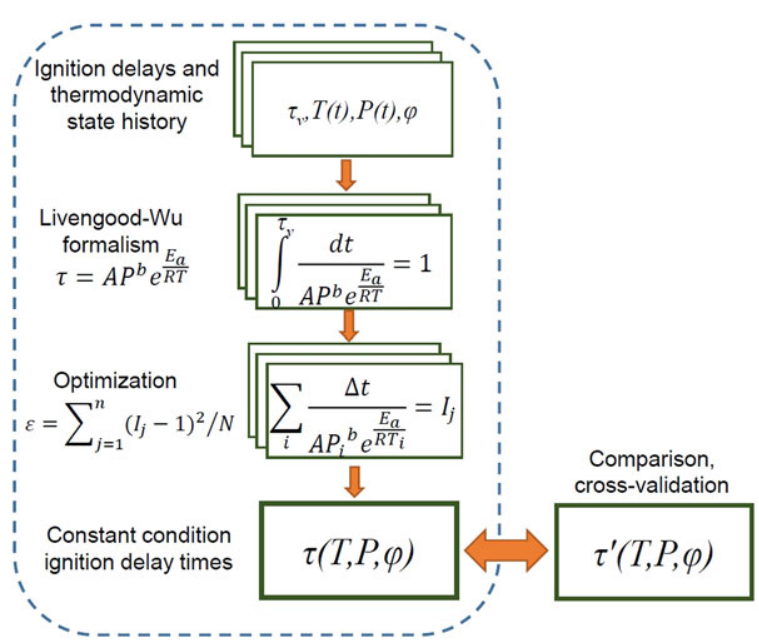

Figure 1 Schematic of the analysis and cross-correlation technique. [Color figure can be viewed at wileyonlinelibrary.com]

for simple fuels and surrogate models of real fuels, due to the large size of the kinetic mechanism or unavailability of the kinetic model for practical multicomponent fuel blends, the conventional and staged L-W methods are still very convenient and powerful tools in ignition and engine knock correlation, especially under complex thermodynamic conditions [12-14].

A direct implication from the $\mathrm{L}-\mathrm{W}$ integral is that if the $P$ and $T$ history for an arbitrary process (e.g., in the HRRST) is known, the consequent ignition delay could be predicted using an ignition delay database or an analytical formula $\tau(T, P)$ accommodating realistic chemistry under constant states. Therefore, conventional shock tube IDT measurements, assuming they also have precisely known $P$ and $T$ histories, could be used to test the consistency with IDT data from the HRRST. Furthermore, a more intriguing inverse process is systematically described in this paper, shown schematically in Fig. 1, where several runs of ignition delay, together with $P(t)$ and $T(t)$ history of each run, can be used in the inverse L-W approach to extract the IDT as an explicit function of constant conditions, i.e., $\tau(T, P)$. A similar idea was also demonstrated by Reyes et al. [15], where the authors tried to obtain the ignition delay under constant conditions based on spark-ignition experiments conducted in a constantvolume combustion bomb. However, their experimental data were largely biased by the low-to-intermediate temperature heat release and NTC, and there was insufficient vigor on the convergence of the algorithm they adopted.

In a high throughput facility, like the HRRST, experiments for IDT can be performed with a considerable reduction in effort compared to a conventional shock tube yet still maintain the desirable characteristics of the shock tube and access the desired thermodynamic conditions. Therefore, even if the thermodynamic state history of an individual experiment is less ideal than a conventional shock tube experiment, effort is spared if the aggregated data contain the same information. In addition, two separate data processing algorithms including both relaxed Newton iteration and genetic algorithm have been demonstrated to control the uncertainty and ensure the global optimal nature of the solution.

The acquired ignition delays must be compared with other published independent data to validate the proposed technique. The measurement of IDT using HRRST, the correlation of HRRST data with reported data from regular ST using the conventional L-W method, and the deconvolution of the high-temperature constant state IDT using the inverse L-W method, are therefore the major objectives of this paper.

\section{EXPERIMENTAL METHODS}

The miniature high repetition rate shock tube (HRRST) at UM-Dearborn has been recently described $[6,16]$. The shock tube (Fig. 2) has a bore of $12.7 \mathrm{~mm}$, and a driven section length of $1.495 \mathrm{~m}$. The HRRST uses the opening of a solenoid driver valve instead of the bursting of a diaphragm (as in conventional tubes) to initiate a normal shock. The shock tube is fully automated and consists of a cycle of filling a charge of test gas, firing the driver valve to initiate the shock wave [17], and then venting the postshock gases. This process can be accomplished at cycle rates up to $\sim 1 \mathrm{~Hz}$ in this miniature shock tube, but typically, and in the experiments presented here, the cycle rate is $0.25 \mathrm{~Hz}$.

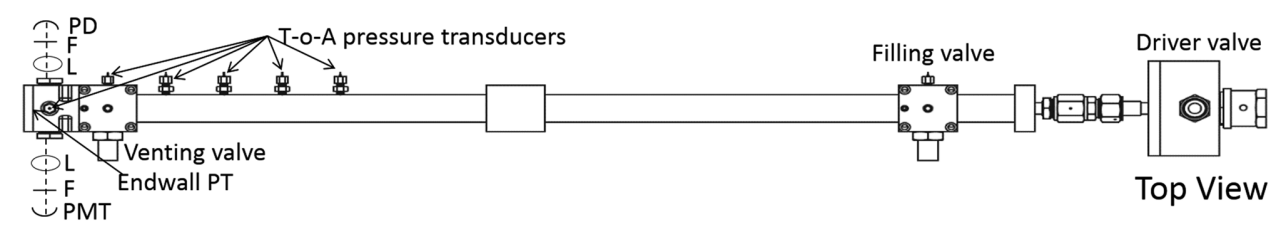

Figure 2 HRRST schematic including optical setup used for experiments. 
All experiments are individually tracked including preshock temperatures and pressures, as well as postshock conditions.

Six wall-mounted Dynasen CA-1135 (Goleta, CA) piezoelectric transducers each fixed $75 \mathrm{~mm}$ apart are used for measuring shock time-of-arrival (i.e. shock velocity). The uncertainty in shock velocity is better than $1 \%$, which results in uncertainty in the calculated reflected shock temperatures (by frozen normal shock conditions) of about $1.2 \%$, or between 10 and $20 \mathrm{~K}$ in reflected shock temperature immediately following reflection. Later, the temperature behind the reflected shock changes, and deviations from the frozen normal shock value are much larger than $10-20 \mathrm{~K}$. At the end wall, a PCB 105C12 (Depew, NY) pressure transducer is mounted to track reflected pressure $P_{5}$. Aside from pressure, chemiluminescence is also measured. The schematic of the diagnostics setup is shown in Fig. 2. Light at $f / 8$, collected $2.5 \mathrm{~mm}$ from the endwall, is sampled through sapphire windows, filtered through $10 \mathrm{~nm}$ bandpass filters $\left(430 \mathrm{~nm}\right.$ for $\mathrm{CH}^{*}$ and $307 \mathrm{~nm}$ for $\mathrm{OH}^{*}$, Andover, Salem, NH), and detected using a photomultiplier tube (Thorlabs PMM01, Newton, $\mathrm{NJ}$ ) for $\mathrm{OH}^{*}$, and an amplified photodiode (Thorlabs PDA100A) for $\mathrm{CH}^{*}$. Signals are digitized at $10 \mathrm{MHz}$ (Measurement Computing PCI-DAS4020/12, Norton, MA) for processing offline using custom scripts written in Labview.

The driver gas used is helium (99.995\%; Purity Plus Gases, Detroit, MI). Test gas mixtures are prepared manometrically in a batch mode into a $15-\mathrm{dm}^{3}$ stainless steel pressure vessel/mixing rig. Reagents include isooctane (99.8\%; Sigma-Aldrich, St. Louis, MO), $n$ heptane (99\%; Sigma-Aldrich), and acetone (99.9\%; Sigma-Aldrich). Reagents are degassed via vacuuminert purge cycles. The oxidizer is oxygen $(99.999 \%$; Purity Plus) and bath gas argon (99.9995\%; Purity Plus). The mixing vessel has a static mixing element, but gases are allowed to set for at least $1 \mathrm{~h}$ before experiments. In all systems, the amount of fuel is kept constant at $1 \%$, but equivalence ratio, $\varphi$, is varied from 0.5 to 1.5 by varying the composition of $\mathrm{O}_{2}$ with balance Ar.

\section{DATA ANALYSIS METHODS}

\section{Estimating Gas State}

Currently, an independent measurement of temperature (i.e., via two-color absorption) is unavailable in the HRRST; however, the temperature trace $T(t)$ can be estimated. One method is based on the isentropic assumption such that the $T(t)$ could be directly estimated from the measured pressure profile. The derivative of temperature in this case is

$$
\frac{d T}{d t}=\frac{d P}{d t} \frac{T \bar{R}}{P \overline{c_{p}}(T)}
$$

where $\bar{R}$ is the universal gas constant and $\overline{c_{p}}(T)$ the constant pressure-specific heat implemented with temperature polynomial taken from Burcat and Ruscic [18]. This estimation is implemented with a simple time marching scheme with experimental information for $d P / d t$ and estimate of $T(t=0)$ from the normal shock relations calculated using the method of Gardiner et al. [19]. The scheme assumes the ideal gas equation of state, but higher pressure studies would also need to incorporate real gas effects [20]. The temperature profile in this case is estimated up to the experimental ignition time. This method is marginally acceptable in the HRRST if (a) the thermodynamic state change is influenced more strongly by gas dynamic effects than by chemical heat release (itself a function of concentrations and conditions) and (b) if the test time is short. Conditions during longer test times might be subject to other problems such as unaccounted for heat loss, other unaccounted for gas dynamic effects, additional uncertainty due to the drift of the piezoelectric transducer, etc. We have also attempted refinements to our estimation of temperature profile based on detailed chemical modeling with validated mechanisms. Those methods perform in a similar fashion as the isentropic assumption used in this work, implying the insignificant role of combined preignition heat release and heat loss, but would not generally be extensible to other systems without validated mechanisms.

\section{Determination of Constant Condition IDT}

In the high-temperature ignition region, the constant condition ignition delay can generally be described by an Arrhenius-like term:

$$
\tau=A P^{n} \exp \left(E_{a} / R T\right)
$$

where $A, n$, and $E_{a}$ are constants to be determined. For this type of kernel, it is straightforward to implement the Livengood-Wu integral, Eq. (1) and discretize in time (subscripted $i$ ) to yield:

$$
1=\int_{0}^{\tau^{\prime}} \frac{d t}{\tau(T(t), P(t))}=\sum_{i} \frac{\Delta t_{i}}{A P_{i}^{n} \exp \left(E_{a} / R T_{i}\right)}
$$


For an ignition test of given mixture with variable thermodynamic conditions, $P(t), T(t)$, and the ignition timing $\tau^{\prime}$ are all known. The main task to acquire the optimized correlation of ignition delay, $\tau(T, P)$, therefore involves the determination of the unknown set of $A, n$, and $E_{a}$, to solve Eq. (4), with least-square errors over the entire domain. It is important to note that the complete domain can be extensive as it is accessed by multiple experiments.

Therefore, for multiple experiments, M, (indexed $m$ ) with different thermodynamic traces leading to different ignition delay times, $M$ residual equations can be formed:

$$
\begin{aligned}
I_{m} & =\left(\sum_{i=1}^{N} \frac{\Delta t_{i}}{A P_{i}^{n} \exp \left(E_{a} / R T_{i}\right)}\right)_{m} \\
& =\sum_{i=1}^{N_{m}} A^{-1} P_{i, m}^{-n} \exp \left(-E_{a} / R T_{i, m}\right) \Delta t_{i, m}
\end{aligned}
$$

And thus the problem is changed into an optimization process seeking the least-squares minimization of the total residual $\varepsilon$ in a certain parameter domain (i.e., $\left.A, n, E_{a} / R\right)$.

$$
\min \varepsilon=\frac{1}{M} \sum_{m=1}^{M}\left(I_{m}-1\right)^{2}
$$

For simplicity, denote $A, n, E_{a} / R$ as $a, b, c$, respectively. Assuming the current values $a^{j}, b^{j}, c^{j}, \varepsilon^{j}$ are known, as are the partial derivatives of $\varepsilon$ with respect to $a, b$, and $c$, the relaxed Newton iterative method can be used to obtain the next values for the parameters, i.e., at step $(j+1)$ :

$$
\left(\begin{array}{l}
a^{j+1} \\
b^{j+1} \\
c^{j+1}
\end{array}\right)=\left(\begin{array}{l}
a^{j} \\
b^{j} \\
c^{j}
\end{array}\right)-\beta\left(\begin{array}{l}
\varepsilon /(\partial \varepsilon / \partial a) \\
\varepsilon /(\partial \varepsilon / \partial b) \\
\varepsilon /(\partial \varepsilon / \partial c)
\end{array}\right)^{j}
$$

where $\beta$ is a relaxation coefficient to ensure the iteration convergence. Iteration stops when convergence of this error function is achieved. In all cases, adopting this algorithm in this study (acetone), it is found that the final values of error function are within 5\%.

It is understood that the relaxed Newton iterative method is highly efficient in finding minima, but may converge to local minima instead of the global minimum. For this reason, it can be sensitive to the initial seed (i.e., $\left.a^{1}, b^{1}, c^{1}\right)$. Because of this sensitivity, we have also used genetic algorithm (GA) [21] to find the $a, b, c$ with a global minimum error $\varepsilon$, i.e. solving Eq. (6) in a wide parameter domain. The GA method for solving optimization problems is based on a natural selection process mimicking biological evolution. In our case, the domain for $a, b, c$ is [1e-11, 2e-10], $[-0.65,-0.35]$, and $[20,000,25,000]$ respectively. GA first randomly selects different combinations (100 in this work) of $a, b$, and $c$ within a domain and then screens those populations performing small errors as the "parents" generation, and modifies the "parents" generation to get the "children" generation including prescribed rules via passing, crossover, and random mutation. Again, iteration stops when convergence of $\varepsilon$ is achieved. For all the three fuels investigated in this work, the final values of $\varepsilon$ almost all fall below $10 \%$, and completely within $15 \%$. The method could be computationally more expensive depending on the parameter domain but can be very useful for finding global minima as described below.

It should be noted that without independent temperature measurement, the problem we address here is an approximate one, which obviously cannot capture the exaggerated temperature change in the HRRST. Therefore, one cannot expect completely quantitative agreement with the conventional shock tube data, and the agreement as achieved in the following is hence considered to be reasonable and satisfactory.

\section{RESULTS AND DISCUSSION}

\section{Ignition Delays in the Miniature HRRST}

Experimental conditions are selected to observe an ignition delay dependence on temperature, pressure, and composition over as wide a range of conditions as possible in the miniature HRRST. The test time in the HRRST is approximately $0.7 \mathrm{~ms}$, although there are some experiments suggesting test times up to $1 \mathrm{~ms}$. Specifically, for each fuel, reflected shock pressures range from $5<P_{5}<11$ bar, and reflected temperatures range from $1050<T_{5}<1600 \mathrm{~K}$. We presently report 2996 experiments, 1310 with isooctane as fuel, 943 with heptane, and 743 with acetone, sufficient for statistical analysis.

Figure 3 shows the typical raw traces from experiments testing for ignition delay time. In this figure, a and $b$ experiments exhibit ignition during the HRRST test time, but $\mathrm{c}$ does not during the HRRST test time. The endwall pressure signal shows some noise, particularly at $t=0$, when the shock arrives and is reflected off of the endwall. This ringing is because the miniature transducer (PCB 105C12) is excited faster than the rise time of the transducer. The underlying signal, however, is reliable. Future refinements could include redesigning the test section to accommodate a larger 
a)

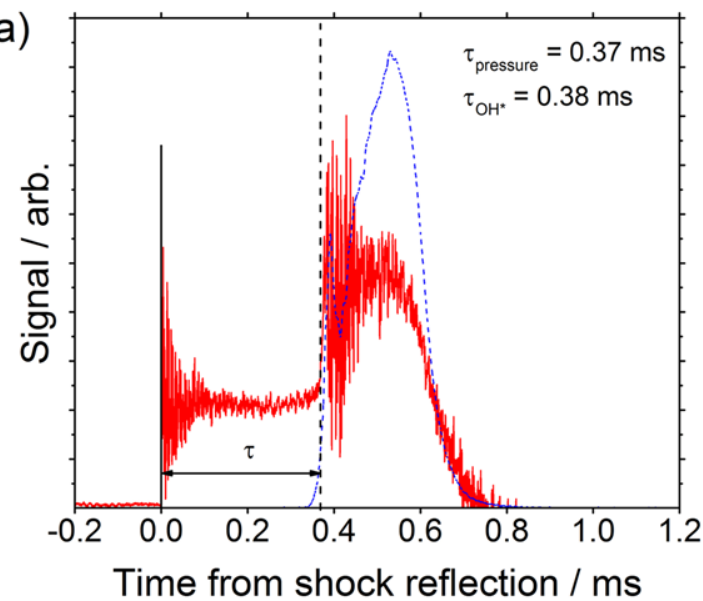

b)

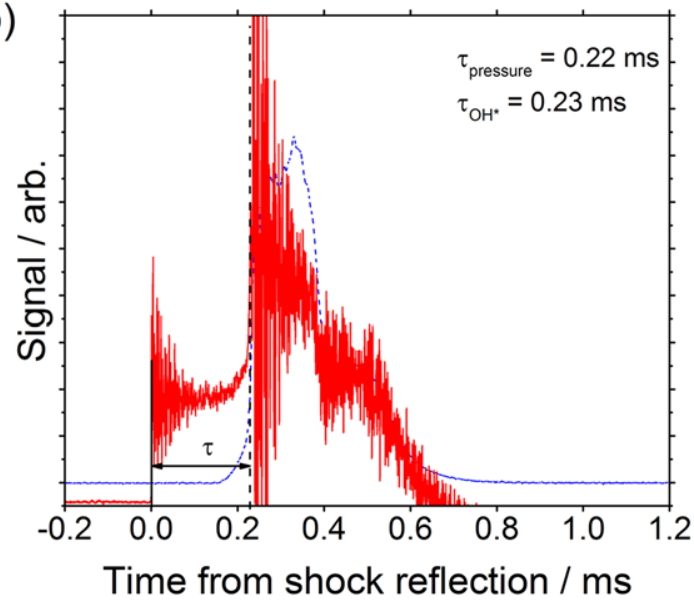

c)

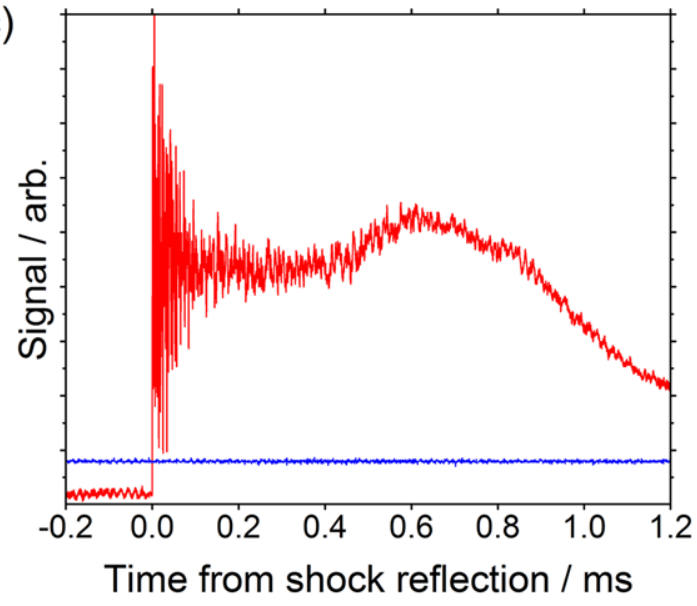

Figure 3 Example experiments in the HRRST. Solid lines are endwall pressure transducer signals, broken lines are $\mathrm{OH}^{*}$ chemiluminescence traces. (a) Ignition delay time measurement: $1 \% \mathrm{i}-\mathrm{C}_{8} \mathrm{H}_{18} / \mathrm{O}_{2} / \mathrm{Ar}, \varphi=1, T_{1}=25.5^{\circ} \mathrm{C}, P_{1}=138$ Torr, $T_{5}=1322 \mathrm{~K}, P_{5}=6.3 \mathrm{bar}$; (b) ignition delay time measurement: $1 \%$ i- $\mathrm{C}_{8} \mathrm{H}_{18} / \mathrm{O}_{2} / \mathrm{Ar}, \varphi=0.5, T_{1}=20.0^{\circ} \mathrm{C}$, $P_{1}=218$ Torr, $T_{5}=1195 \mathrm{~K}, P_{5}=9.3 \mathrm{bar}$; (c) experiment which does not ignite during test time: $1 \% \mathrm{i}-\mathrm{C}_{8} \mathrm{H}_{18} / \mathrm{O}_{2} / \mathrm{Ar}, \varphi$ $=1.5, T_{1}=28.1^{\circ} \mathrm{C}, P_{1}=194$ Torr, $T_{5}=1104 \mathrm{~K}, P_{5}=6.0$ bar. [Color figure can be viewed at wileyonlinelibrary.com] pressure transducer to eliminate the ringing. After arrival of the reflected shock, there is a relatively flat pressure profile (approximately $250 \mu \mathrm{s}$ in Fig. 3a, less than $200 \mu \mathrm{s}$ in Fig. 3b) followed by a gradual rise until the ignition event. The reflected shock pressure trace $P(t)$ measured with a pressure transducer in the HRRST reveals that the thermodynamic condition is varying during each test. The change in pressure suggests that the temperature in the conditions behind the reflected shock increases during the test time (including before ignition).

There are at least four effects contributing to any pressure change in the HRRST. First, the pressure increases as the reflected shock propagates back into the incident shock region with growing sidewall boundary layers. This pressure rise would be present in any shock tube, but the small bore of the HRRST exacerbates the effect compared to conventionally sized shock tubes. In the HRRST, because of the short length, this pressure increase merges with and can be hard to discern from the second effect [6], the late hump (and subsequent decay) dominated by the contact surface and rarefaction fan [22]. These types of pressure signals are observed even in conditions without strong ignition events (e.g., Fig. 3c) and are observed even with inert test gases $[6,16]$. In reacting conditions, the third effect is the pressure changes with the change in temperature and density during the reactions leading to the strong ignition event (i.e., preignition chemistry and induced heat release). The fourth effect is the ignition event, which produces strong waves and again rings the transducer. The temperature behind the reflected shock is not measured but modeled as described in the section Estimating Gas State. In terms of the assumptions behind calculating the temperature profile, we assume the first and second effects dominate the third effect up to the ignition delay time. This is a reasonable assumption based on comparison to the profiles in experiments that do not exhibit ignition, e.g., Fig. 3c. Furthermore, we assume that we are able to discern the ignition event and model the preignition pressure trace uncontaminated by ignition. This is a very good assumption in the conditions of this study (chosen for strong high-temperature ignition), but could be a future limitation. Significant effort has been expended to develop robust automated routines for identifying the ignition delays via either the pressure trace or the chemiluminescence trace as well as to fit a polynomial coefficient to the preignition pressure trace for efficient processing using the methods of given in the section Data Analysis Methods. More details on this can be found in Ref. 23. We report ignition delays based on time from shock reflection to the location of maximum gradient in pressure or chemiluminescence traces. We 
have also tried other criteria such as a simple percent of maximum threshold (example $15 \%$ of chemiluminescence signal), back extrapolation to baseline from maximum gradient, etc. (in the Supplemental Information). There are only very small differences in values, trends with temperature, or scatter.

As temperature is the strongest factor for ignition delay time, for each fuel, the ignition delay times are plotted in logarithm scale and against the inverse temperature in Fig. 4. The inverse temperature in this plot is calculated from the frozen chemistry temperature behind a reflected normal shock. The ignition delay trends are qualitatively similar to those reported in previous studies $[24,25]$. For a given pressure and equivalence ratio, there is a clear dependence on inverse temperature indicative of Arrhenius-like behavior describing the high-temperature ignition of these fuels. The isooctane and acetone ignition delays show little scatter in sequential measurements at the same conditions, yet they are very sensitive to incremental changes in conditions. The data show more scatter for ignition delays approaching $1 \mathrm{~ms}$ because these ignition events are happening very late in the test time of HRRST including those on the downward slope after the peak in pressure but still showing ignition before the test time (taken as the time at which the pressure drops to $80 \%$ of the peak pressure [20]).

Ignition delay times with $n$-heptane as fuel show slightly more scatter than those with iso-octane or acetone. There are likely two reasons for this. First, the signal levels for ignition events with $n$-heptane (both pressure transducer traces and chemiluminescence traces) were the lowest of the three fuels. Second, the $n$ heptane experiments, particularly at low temperature and high pressure are the most likely of these fuels to exhibit low-temperature ignition behavior. Such deviations at low temperature are also observed in Figs. 7 and 8 in the next section. While the pressure transducer trace does not exhibit strong secondary humps, it is possible that these are masked by the other pressure effects as described above. On occasion, the chemiluminescence traces show double peaks. Our current processing scheme does not distinguish low-temperature heat release or complicated two-stage ignition behavior should it occur, but future studies would attempt to resolve this.

In assessing the uncertainty in experimental IDTs (i.e., HRRST IDTs), the uncertainty in reported ignition delay time is small. The rise time of the pressure transducer is $<2 \mu \mathrm{s}$. In all cases, the time at which the reflected shock reaches the endwall is determined to this level unambiguously. These experimental conditions are selected such that the onset of ignition via both the chemiluminescence signal and pressure trans- a)

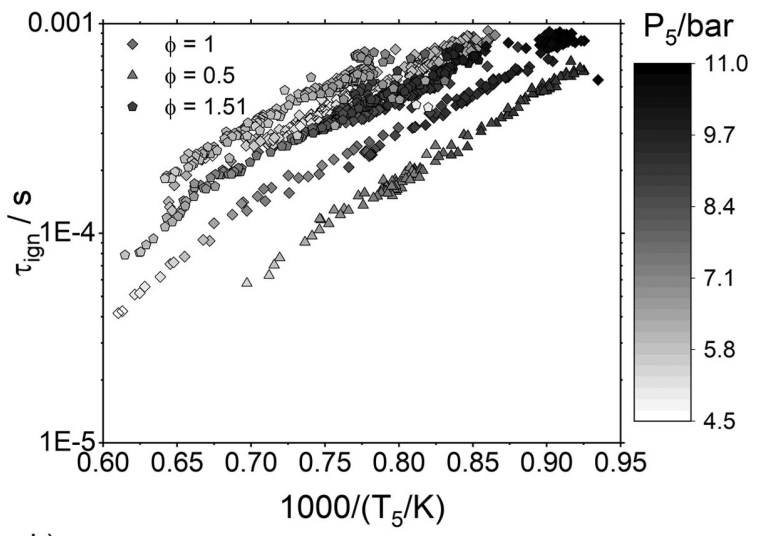

b)

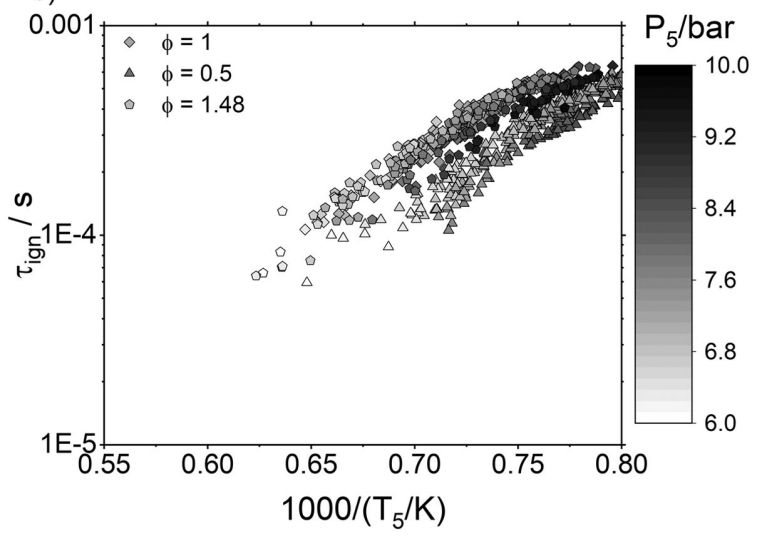

c)

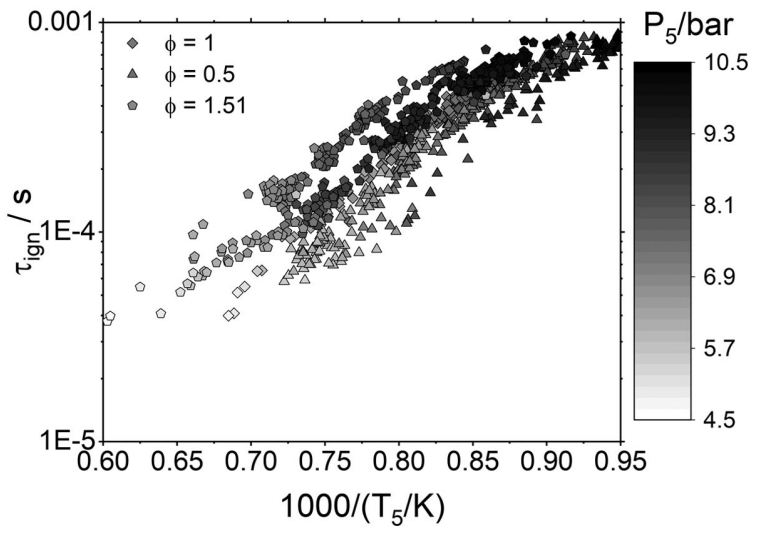

Figure 4 Ignition delay measurement versus reflected shock temperature $T_{5}$ for different pressures and equivalence ratios. Mixtures are $1 \%$ fuel $/ \mathrm{O}_{2} / \mathrm{Ar}$. (a) Iso-octane, (b) acetone, and (c) $n$-heptane.

ducer signal are also unambiguous. The rise time of the photomultiplier tube (PMT) used was $15 \mu \mathrm{s}$. For most experiments, this uncertainty represents a small fraction of the assigned ignition delay time. There are $<20$ of the experiments with an assigned ignition delay below $50 \mu \mathrm{s}$. These do have greater relative uncertainty in ignition delay time but represent a very small 
fraction of the data used in this analysis. Future refinements to the experimental apparatus can include faster PMTs. Overall, the IDTs determined by the chemiluminescence signal and pressure transducer signal agree within 5\%, and more typically within 3\% (as in Fig. 3). They are highly repeatable in most cases (as in Figs. 4a and $4 b$ ). Therefore, it is thought that the uncertainty in experimental IDT is limited by the rise time of the transducers, and the determination of the IDT via the automated solver does not contribute greatly to the uncertainty in IDT for a specified IDT criterion, nor does the IDT criterion contribute significantly to the uncertainty in IDT for these experiments.

On the other hand, the greater uncertainty should be considered as lying with the experimental conditions. As described previously, the uncertainty in the frozen chemistry reflected shock temperature is about $1.2 \%$. The uncertainty in the frozen reflected shock pressure is about $3 \%$. The uncertainty in the frozen chemistry reflected shock pressure is higher because there is about $1 \%$ uncertainty in initial test gas pressure, compared to $<0.3 \%$ uncertainty in initial test gas temperature. While a $3 \%$ uncertainty in pressure has a relatively small effect on the ignition delay, because of the exponential nature of the process, $1.2 \%$ uncertainty in temperature should have an effect. Later, the temperature behind the reflected shock changes, and deviations from the frozen normal shock value are much larger than $1.2 \%$ with greater uncertainty.

\section{Direct Comparison of Ignition Delay Times not Accounting for State History}

The voluminous set of ignition delay times can be used to develop correlations for direct comparison to previous experimentally obtained ignition delay times. For example, for iso-octane $/ \mathrm{O}_{2} / \mathrm{Ar}$ mixtures, the ignition delays from Fig. 4a can be described by an Arrheniuslike correlation via least squares fitting the experimental data:

$$
\frac{\tau_{i g n}}{m s}=0.037\left(\frac{P_{5,0}}{b a r}\right)^{-0.7287} \varphi^{0.7376} e^{\frac{7812 K}{T_{5,0}}}
$$

This correlation ought to be considered a naïve correlation and is not recommended for use. The correlation is based on the initial temperatures and pressures behind the reflected shock $\left(T_{5,0}, P_{5,0}\right)$ and does not account for the changing temperature and pressure history in HRRST. Figure 5a shows the agreement between the correlation and the experimental data, which would be considered fair, but probably could be further improved in the highest temperature range.
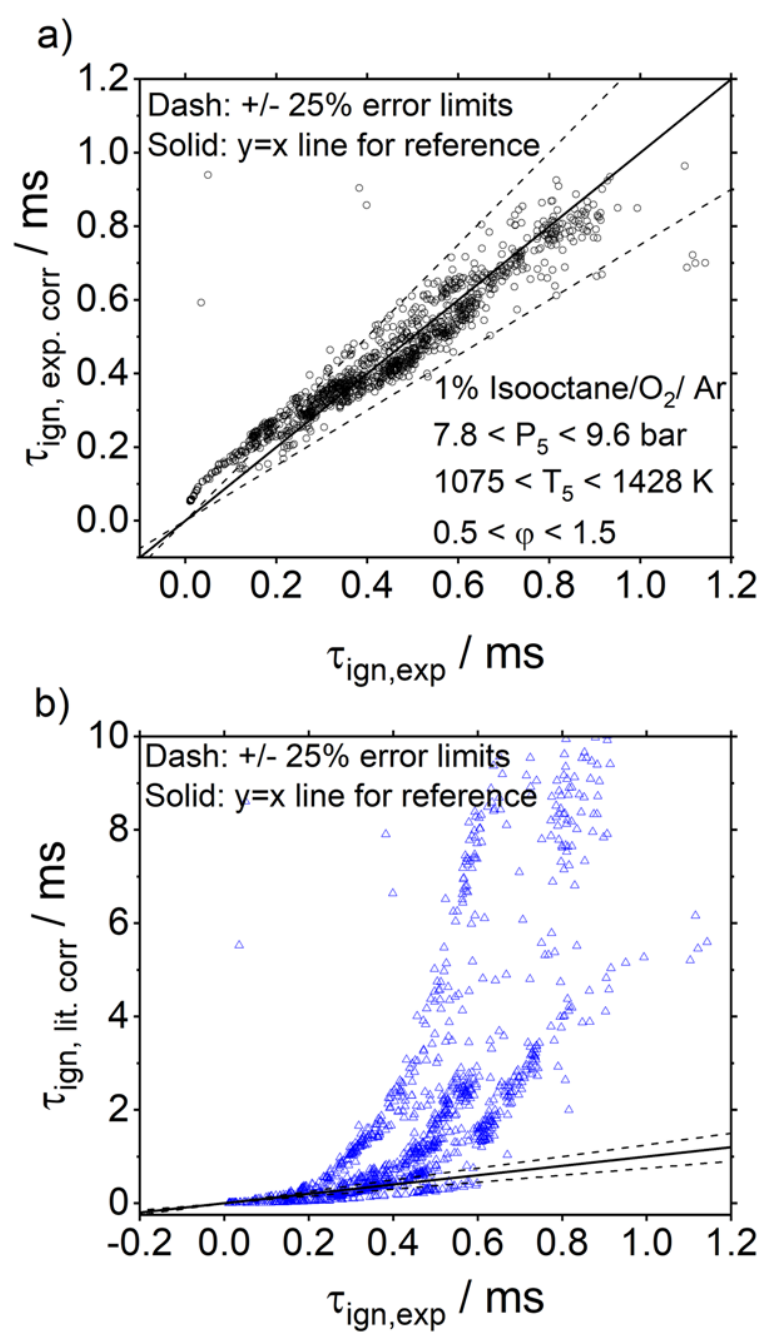

Figure 5 Example of correlation of ignition delay from reflected frozen shock conditions for iso-octane. (a) The correlation to experimental data with naïve consideration of temperature and pressure conditions. (b) The correlation found by Oehlschlaeger et al. [25] [Color figure can be viewed at wileyonlinelibrary.com]

Figure $5 \mathrm{~b}$ compares the experimental data to the prediction of a correlation created by Oehlschlaeger et al. [25] developed from ignition delay studies in a conventional shock tube. The agreement between IDT in the HRRST and the correlation is better for short IDTs than for longer IDTs but overall poor. The most important parameter again is the temperature, suggesting that some higher HRRST temperature input into the correlation of Oehlschlaeger et al. would fit the correlation better than the initial temperature behind the reflected shock. This intuition would match the discussion of the section Ignition Delays in the Miniature HRRST. A $\pm 1.2 \%$ temperature shift indicated by the uncertainty in reflected shock frozen temperature 
is insufficient to cover all conditions. At first glance, it might seem possible to refit some higher average temperature to an observed ignition delay time. Corrections of this nature are sometimes encountered in simpler systems (e.g., single pulse shock tubes employing either chemical thermometry or internal reactive standards $[26,27])$; however, this is an inappropriate method in this case. On the other hand, the underestimation of ignition delays using Eq. (8), compared to those found by Oehlschlaeger et al., implies the crucial effect from the varying thermodynamic conditions in HRRST.

While the temperature is not currently independently measured, it can be estimated using the above assumed temperature profiles described in the section Estimating Gas State from the measured reflected shock pressure profile. One typical example of this is shown in Fig. 6. Using the model estimation, the temperature appears to rise about $80 \mathrm{~K}$ before ignition. The general trend appears to be correct, and we have confidence in the temperature increase. We are not entirely convinced that the temperature trend is nonmonotonic as shown in Fig. 6b. This might be an artifact of using a polynomial fit of pressure that increases with time, but is nonetheless very flat at early times when calculating Eq. (2). In an ignition system, multiple reactions contribute each with different Arrhenius-like terms, whereas in a chemical thermometry study there is only a single reaction. Therefore, in a system with varying temperatures during the test time, there is a large uncertainty from unaccounted for interaction effects or secondary chemistry. The result is that some refinement based on average temperature would unevenly affect regions with seemingly good agreement with the Oehlschlaeger correlation, i.e. short test times with pressure profiles that are very flat. Rather than a direct comparison, or some comparison with an average temperature correction, a better comparison is demonstrated by accounting for the variation of state history of the miniature shock tube $\left(T_{5}(t), P_{5}(t)\right)$ in the following.

\section{Ignition Data Consistency Checking Using Forward L-W}

It is straightforward to test the consistency of the measured ignition delay time in HRRST with those published data from regular shock tubes, using the conventional (i.e.. forward) L-W correlation, Eq. (1) with the measured pressure profile and the estimated temperature profile in the HRRST. The following correlations have been reported for the high-temperature ignition delay of iso-octane, $n$-heptane, and acetone, respectively, with $\mathrm{Ar}$ as inert gas:
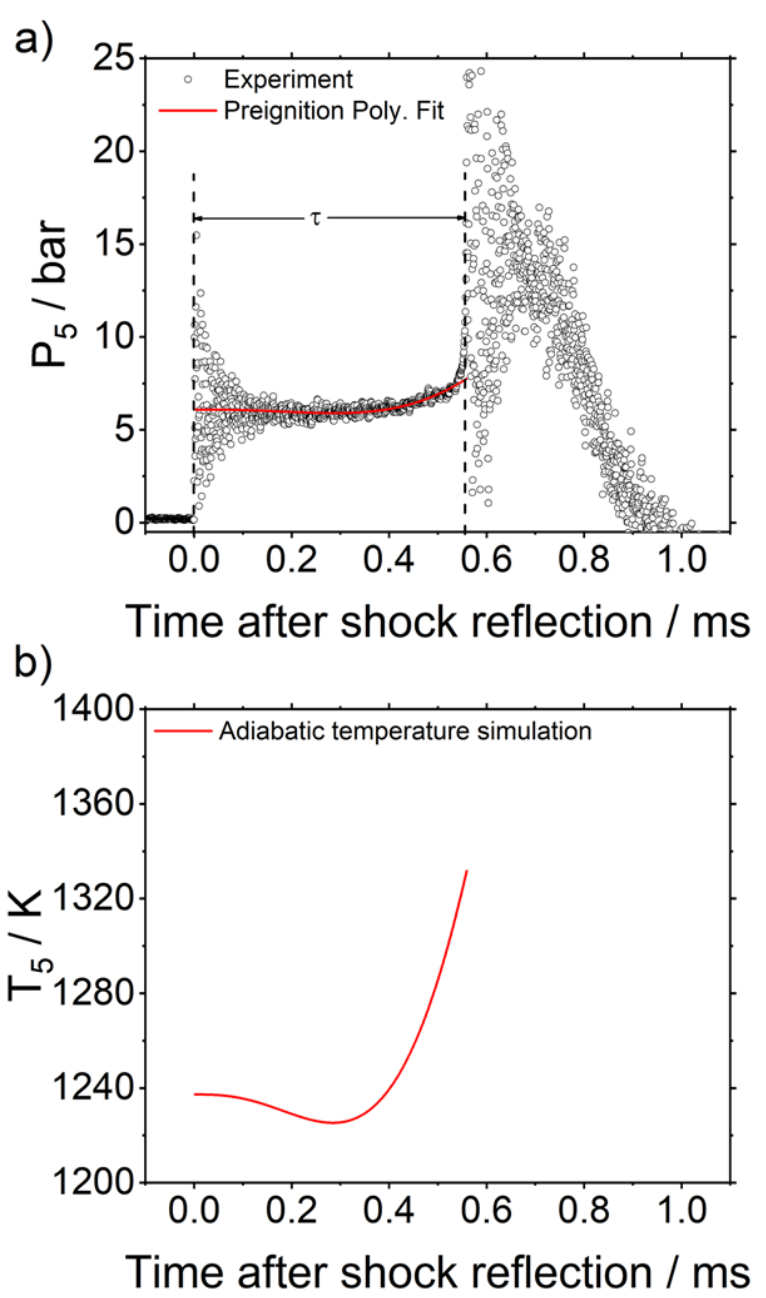

Figure 6 Example of the pressure fitting and temperature estimation as described in the text. $1 \% \mathrm{i}-\mathrm{C}_{8} \mathrm{H}_{18} / \mathrm{O}_{2} / \mathrm{Ar}, \varphi=$ $1, T_{1}=25.9^{\circ} \mathrm{C}, P_{1}=166$ Torr, $T_{5}=1237 \mathrm{~K}, P_{5}=6.7$ bar. [Color figure can be viewed at wileyonlinelibrary.com]

iso-octane from Davidson and Hanson (hereafter referred as Stanford data) [28]:

$$
\begin{aligned}
\tau_{i g n}= & 1.65 \times 10^{-10} P^{-0.66} X_{O 2}{ }^{-0.34} \phi^{1.13} \\
& \times \exp \left(\frac{43050}{R T}\right) \exp \left(-129 X_{\text {fuel }}\right)
\end{aligned}
$$

$n$-heptane from the same source [28]:

$$
\begin{aligned}
\tau_{i g n}= & 6.67 \times 10^{-12} P^{-0.61} \\
& \times X_{O 2}{ }^{-0.68} \phi^{0.96} \exp \left(\frac{44600}{R T}\right)
\end{aligned}
$$




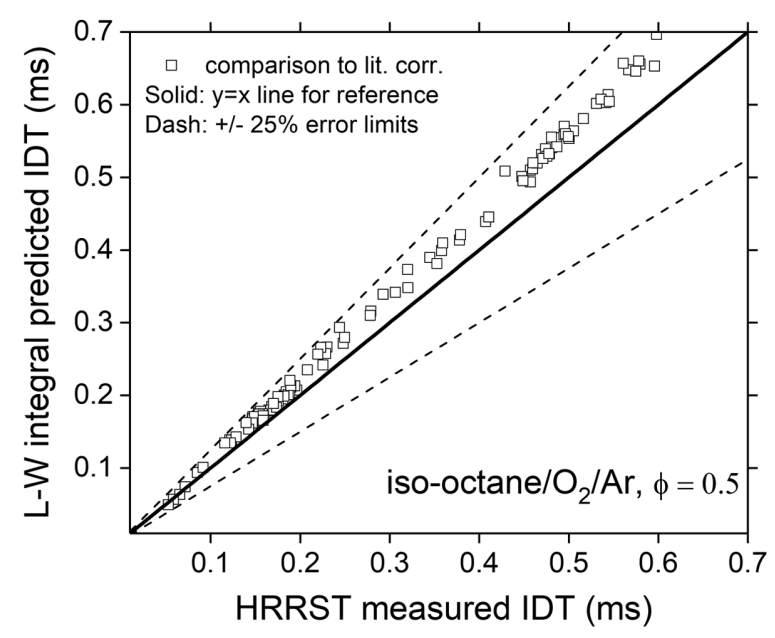

Figure 7 Comparison of ignition delay measured in HRRST and the L-W correlation using published ignition delay data from regular shock tubes (Davidson and Hanson [28]) for $1 \%$ iso-octane/ $\mathrm{O}_{2} / \mathrm{Ar}$ mixture with $\varphi=$ 0.5 , measured pressure profile, and estimated temperature profile as described in the text.

and acetone from Pichon et al. (hereafter referred as NUI Galway data) [29]:

$$
\begin{aligned}
\tau_{i g n}= & 4.75 \times 10^{-13}\left[\left(\mathrm{CH}_{3}\right)_{2} \mathrm{CO}\right]^{0.760 \pm 0.065} \\
& \times\left[\mathrm{O}_{2}\right]^{-1.269 \pm 0.051} \exp \left(\frac{21065 \pm 434}{T}\right)
\end{aligned}
$$

where $\tau_{\text {ign }}$ has units of $\mathrm{s}, T$ has units of $\mathrm{K}, P$ has units of atm, $R$ has units of cal $\mathrm{mol}^{-1} \mathrm{~K}^{-1}$, and the concentrations of fuel and oxygen have units of $\mathrm{mol} \mathrm{cm}^{-3}$, and $X$ is mole fraction. To test the sensitivity of the $\mathrm{L}-\mathrm{W}$ correlation, acetone ignition delay data from Davidson et al., [30] (hereafter also referred to as Stanford data) has also been adopted as an additional comparison. While there are certainly other available correlations and ignition delay data for these three fuels, these were considered to derive from relatively constant condition experiments in conventional shock tubes.

In Figs. 7 and 8, direct comparisons of the HRRST measured ignition delay and the L-W method using published ignition delay correlations from regular shock tubes have been made for two mixtures including iso-octane $/ \mathrm{O}_{2} / \mathrm{Ar}$ and $n$-heptane $/ \mathrm{O}_{2} / \mathrm{Ar}$ with the same equivalence ratio $\varphi=0.5$. Measured pressure traces and assumed temperature traces as described above have been applied to the correlations with Eq, (1) and compared against the experimentally obtained HRRST IDT. Clearly, most of the data are well within the $\pm 25 \%$

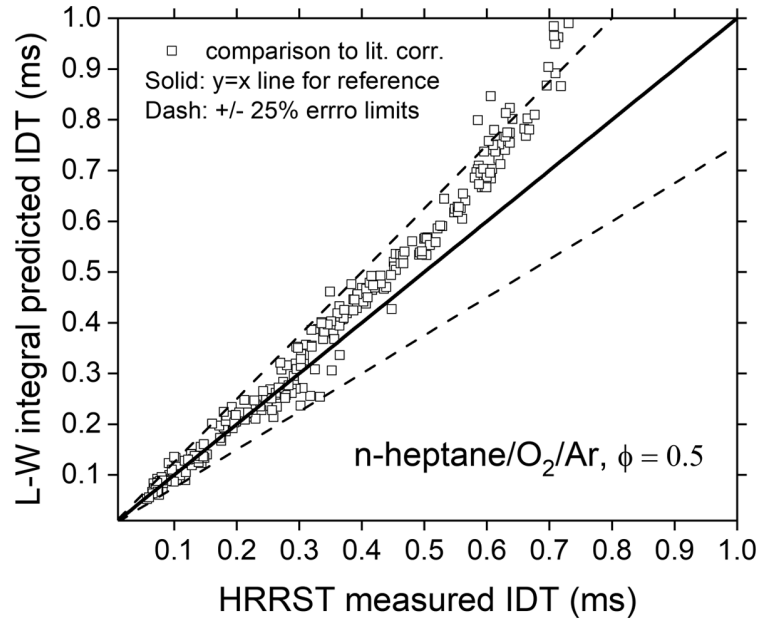

Figure 8 Comparison of ignition delay measured in HRRST and the L-W correlation using published ignition delay data from regular shock tubes (Davidson and Hanson [28]) for $1 \% n$-heptane $/ \mathrm{O}_{2} / \mathrm{Ar}$ mixture with $\varphi=$ 0.5 , measured pressure profile, and estimated temperature profile as described in the text.

error range and distributed along the unity line for reference, demonstrating good correlation and consistency between ignition delays measured in HRRST and regular shock tubes. It seems from these plots that the ignition delays measured in the HRRST are systematically faster than those predicted by the L-W integral method and correlation data in some conditions. The most likely explanation is that the temperature profile based on isentropic assumptions (and the polynomial fit to the pressure profile) used for the L-W correlation is uncertain and perhaps systematically underestimates the true temperature in these conditions. There are several reasons this could happen as discussed in the section Estimating Gas State, including preignition heat release, late drift in the pr,essure transducer, etc. Another possibility is uncertainty in the reported IDT correlations used as the kernel for the L-W correlation. For example, some authors report and account for the normalized pressure change rate $(d P / P) / d t$ during ignition delay experiments, which has been shown to be important in interpretation [31]. An independent temperature measurement in the future will distinguish these possibilities.

In Fig. 9, the comparison of ignition delay for acetone $/ \mathrm{O}_{2} / \mathrm{Ar}$ mixture with $\varphi=1.0$ with fuel mole fraction $1 \%$ is shown. The IDT measured in the HRRST are compared against those predicted using the L$\mathrm{W}$ correlation (as above) based on two independent published constant condition IDT correlations from conventional sized shock tubes. Apparently, the NUI 


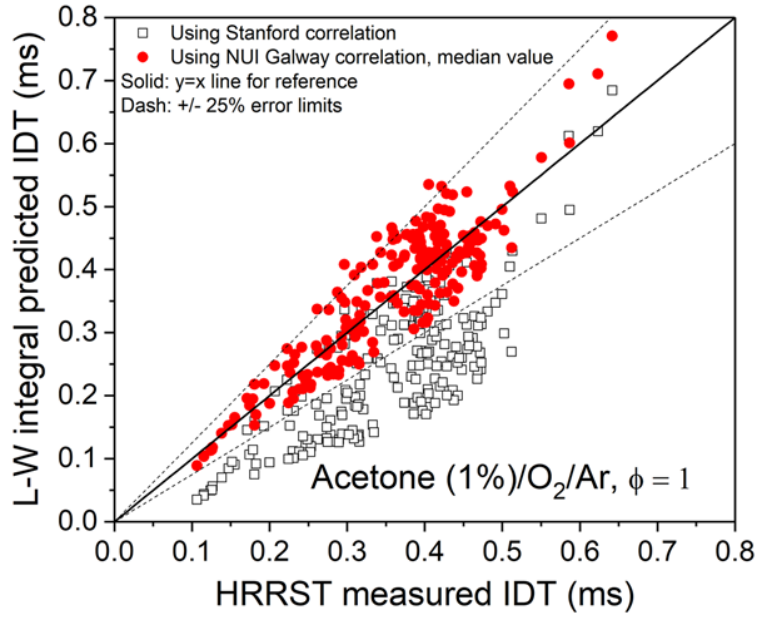

Figure 9 Comparison ignition delay measured in HRRST and the L-W correlation using independently published IDT from regular shock tubes for $1 \%$ acetone $/ \mathrm{O}_{2} / \mathrm{Ar}$ mixture with $\varphi=1.0$, measured pressure profile, and estimated temperature profile as described in the text. [Color figure can be viewed at wileyonlinelibrary.com]

Galway ignition delay data has better correlation with the HRRST data, with agreement generally within the $\pm 25 \%$ error range and distributed fairly evenly around the unity line. On the other hand, the Stanford data has a poorer correlation with the HRRST data, namely the Stanford data lie about $25-50 \%$ faster than the HRRST data. This comparison implies a small difference in the published ignition delay using conventional shock tubes, but a level of uncertainty typically encountered in ignition delay or elementary rate constant measurements. Because of the current uncertainties in temperature and scatter in the correlations, it is difficult to claim with certainty far better agreement with one correlation over the other for these similar correlations. We have nevertheless determined that our ignition delay determination criterion (which is the same as that in Pichon et al. [29]) was not enough to make the difference between better agreement with the NUI Galway correlation vs. the Stanford data.

\section{Constant State IDT De-convolving Using Inverse $\mathbf{L}-\mathbf{W}$}

The success of the conventional L-W in the correlation of HRRST data to regular shock tube data adds more confidence to its inverse problem. Starting with the case shown in Fig. 10, the inverse L-W was applied to HRRST data, using the relaxed Newton iteration, i.e., Eqs. (5)-(7). Two initial seeds are used to initiate

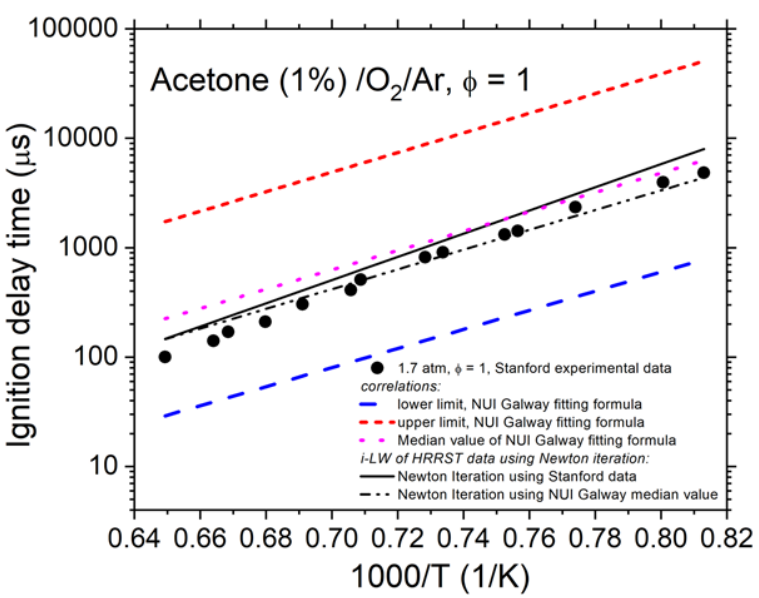

Figure 10 Demonstration of inverse L-W results for $1 \%$ acetone $/ \mathrm{O}_{2} /$ Ar mixture with $\varphi=1.0$, based on HRRST measured ignition delays, measured pressure profile and estimated temperature profile as described in the text. This figure shows comparison to Stanford and NUI Galway data. Independent data sets for IDT have been used to initiate the relaxed Newton iteration. [Color figure can be viewed at wileyonlinelibrary.com]

the error function as shown in Fig. 10. One is based on the Arrhenius fitting of the Stanford data, and the second is based on the median value of NUI Galway correlation. It can be seen in Fig. 10 that the optimized solutions (with error tolerance around $2 \%$ ) from both initial seeds approach similar results. For comparison, we have plotted the experimental IDTs from Stanford, scaled to $1.7 \mathrm{~atm}$ (using their recommended pressure exponent). The correlation from NUI Galway is also plotted. As expected, the optimizations approach both sets of data as they are known (from the section Ignition Data Consistency Checking Using Forward L-W) to provide fair agreement for correlation of IDTs using forward L-W. Depending on initial seed, the converged solution for IDTs lies a little below the NUI Galway results at low temperature and perhaps a bit higher than the Stanford data at high temperature. These results show the feasibility of deconvolving constant state ignition delay using the HRRST and the inverse L-W method, and further show correlation between the current HRRST data and existing experimental data, probably closer to the NUI Galway data.

While the relaxed Newton method is computationally efficient, it does demonstrate some apparent local minima, which would lead to uncertainty given the choices of initial seed. To evaluate the effect of initial guess, two randomly selected initial seeds spanning orders of magnitude of IDT beyond the upper and lower limits of the NUI Galway correlation have been selected to start the inverse L-W iteration based 


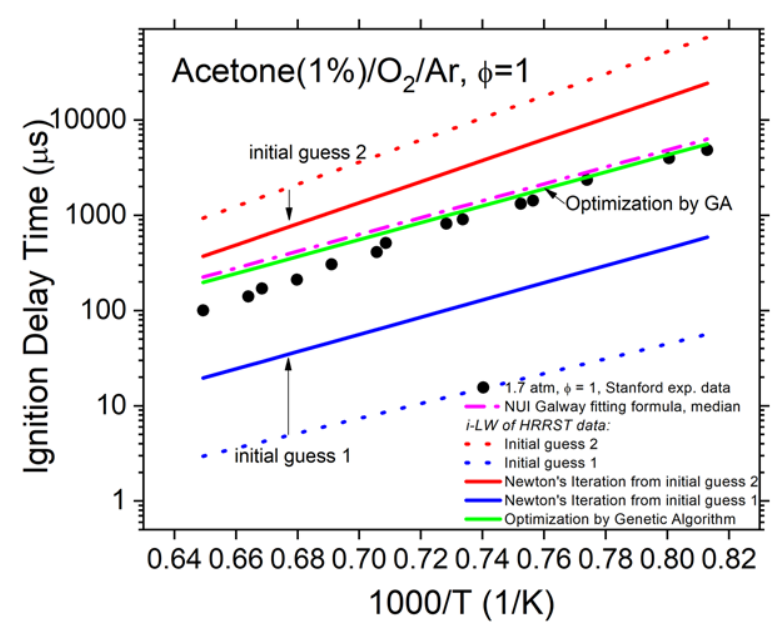

Figure 11 Further demonstration of inverse L-W results as in Fig. 10, with two randomly selected initial values for relaxed Newton iteration and an optimization result by the GA method as described in the text for acetone/ $\mathrm{O}_{2} / \mathrm{Ar}$ mixture with $\varphi=1.0$ with mole fraction of fuel $1 \%$. [Color figure can be viewed at wileyonlinelibrary.com]

on relaxed Newton iteration. Specifically, for initial guess $1, A, n$, and $E_{a} / R$ take the values of $4 \mathrm{E}-11,-0.9$, and 18,000 respectively, whereas in the case of initial guess 2 , the corresponding values are $3.8 \mathrm{E}-11,-0.6$, 26,700, respectively. As shown in Fig. 11, optimized solutions obtained by the Newton iteration do approach the experimental data sets from either direction, but apparently find local minima. While an infinite number of arbitrary guesses could be used as initial seeds for the Newton iteration based method, the apparent differences between the two localized optimal solutions nevertheless suggest the necessity of some global optimization strategies.

To completely avoid the effect of initial guess, the GA method is also used to refine the optimized ignition correlation among local solutions in a wide parameter space, as described in the section Determination of Constant Condition IDT. It is seen in Fig. 11 that the global optimization based on GA shows good agreement with the relaxed Newton results when initiated from closer seeds (in Fig. 10), and both are fairly close to the experimental data in conventional shock tubes for comparison. For this case, the final $\varepsilon$ value of the GA method is less than $2 \%$. Also, it is worth noting that, compared to a certain relaxed Newton iteration with initial guess falling into the range of the GA method, the GA method ends up with the same error in the worst case, and it always generates better solutions due to its intrinsic global optimization feature.

With the variation of thermodynamic states accounted for using the inverse L-W method, as shown
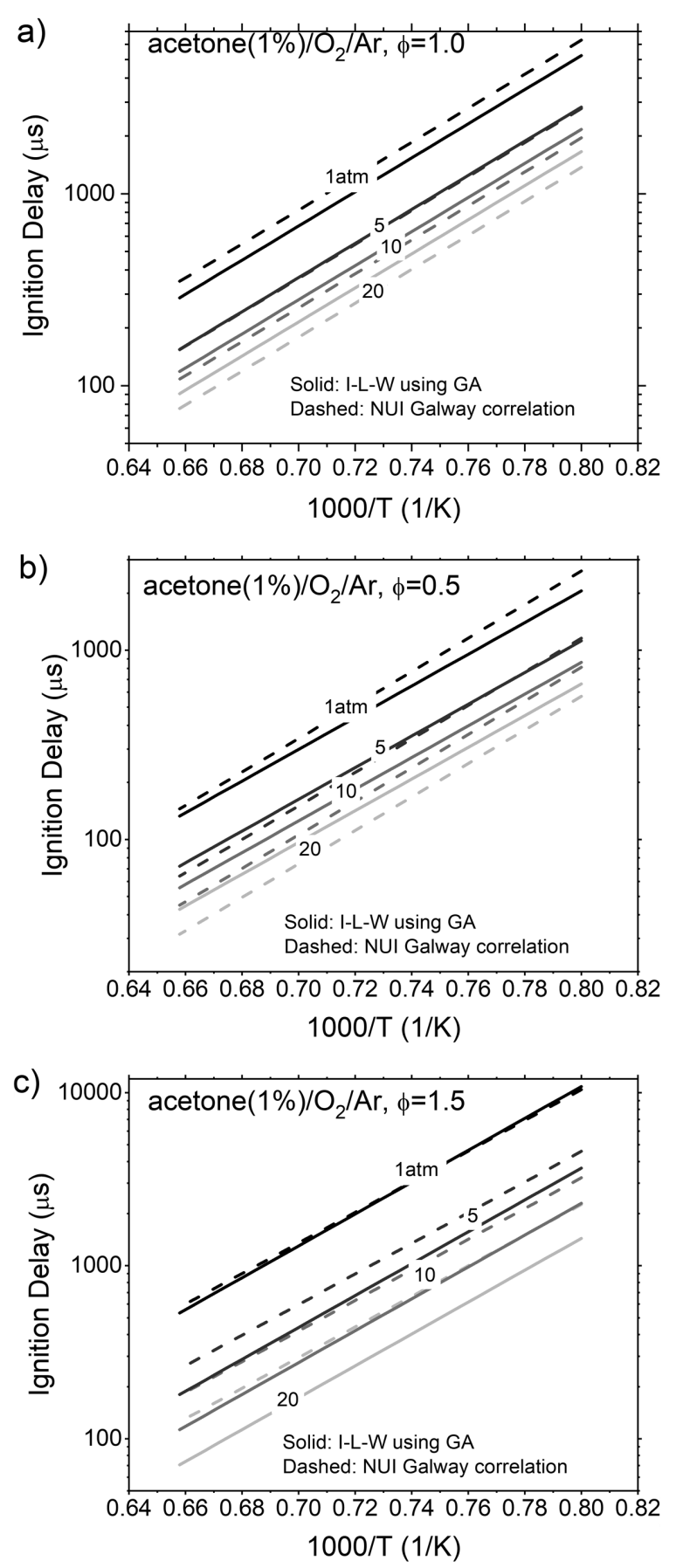

Figure 12 Comparison between inverse L-W results of HRRST using GA methods with those from NUI Galway regular shock tube, for acetone $(1 \%) / \mathrm{O}_{2} / \mathrm{Ar}$ mixture with (a) $\varphi=1.0$, (b) 0.5 , and (c) 1.5 .

in Figs. 12-14, satisfactory agreement has been found between the ignition delay time deconvolved from HRRST using the GA-based inverse L-W method, and those from experimentally determined correlations under different temperature, pressure, and thermodynamic states from the literature. There are 


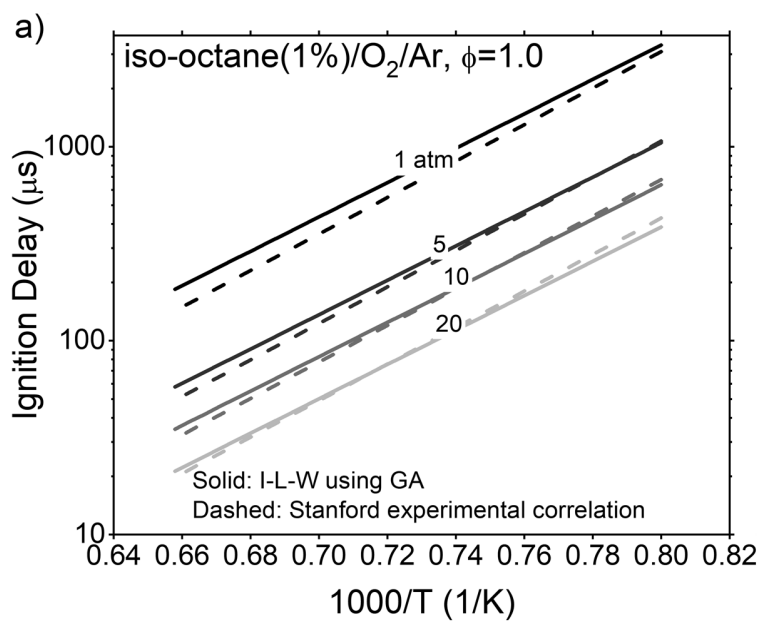

b)

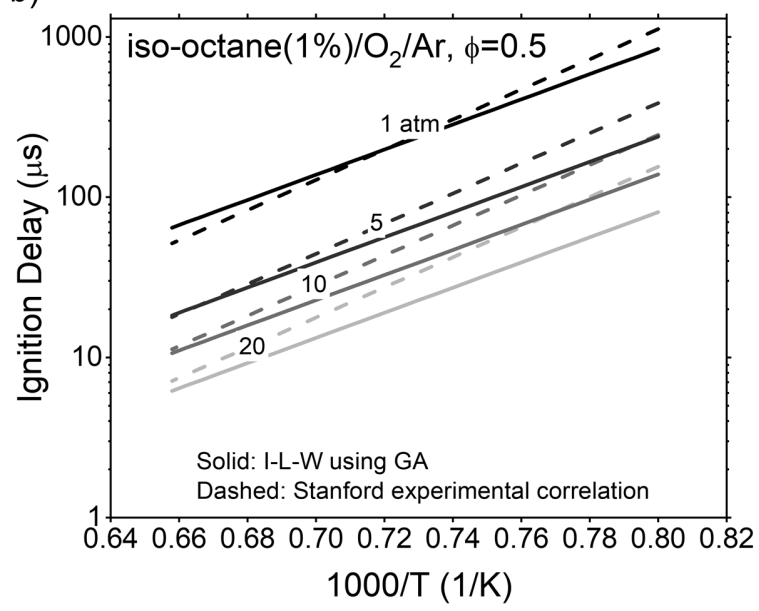

c)

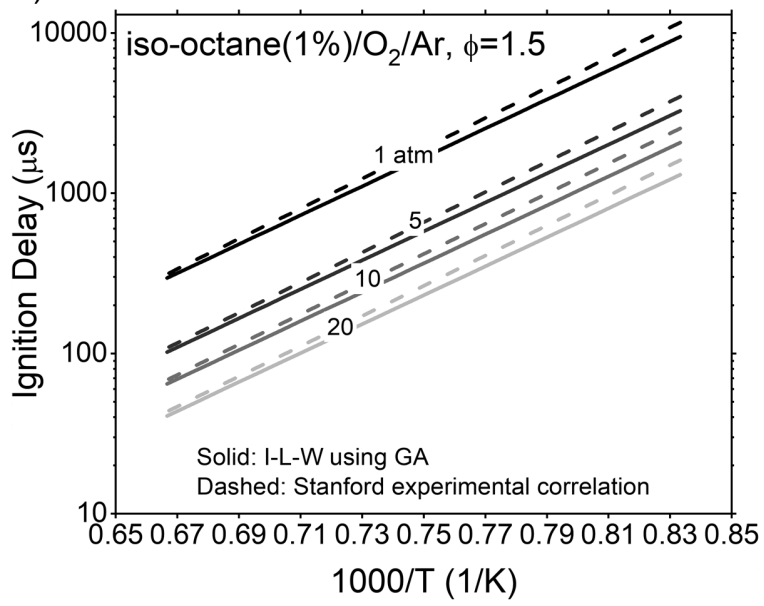

Figure 13 Comparison between inverse L-W results of HRRST using GA methods with those from Stanford regular shock tube, for iso-octane $(1 \%) / \mathrm{O}_{2} / \mathrm{Ar}$ mixture with (a) $\varphi=1.0$, (b) 0.5 , and (c) 1.5 .
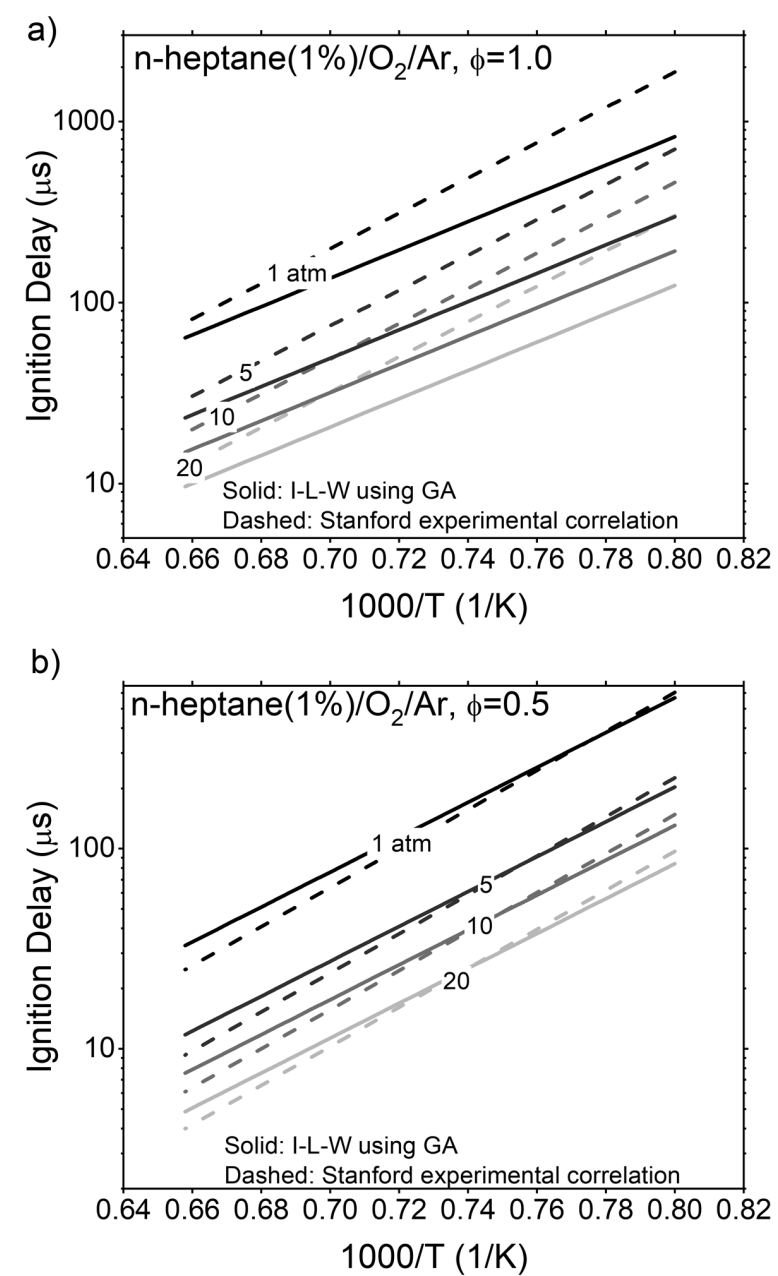

c)

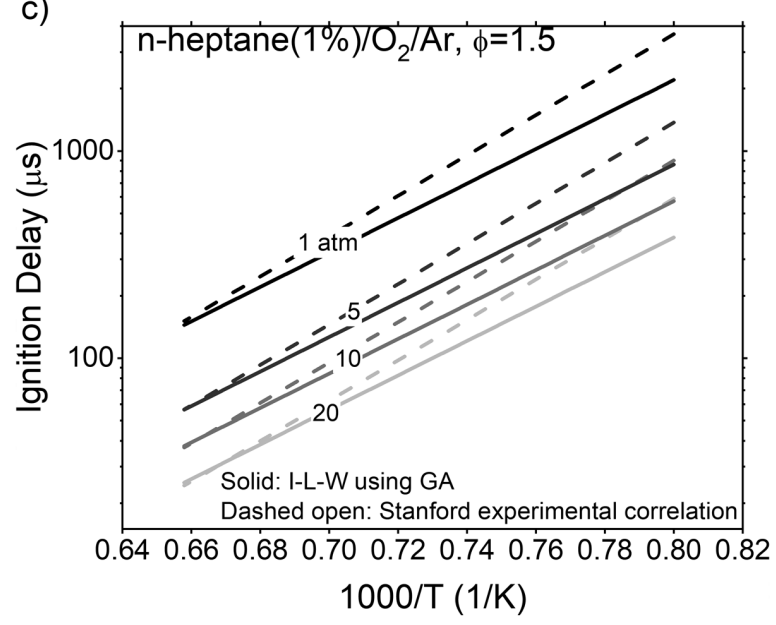

Figure 14 Comparison between inverse L-W results of HRRST using GA methods with those from Stanford regular shock tube, for $n$-heptane ( $1 \%) / \mathrm{O}_{2} / \mathrm{Ar}$ mixture with (a) $\varphi=1.0$, (b) 0.5 , and (c) 1.5 . 
a few conditions for which the agreement is poorer. For example, for the very fast high temperature ignition delays in $n$-heptane: this region has the highest uncertainty in the measured ignition delay time, and the experimental HRRST data in this region are very sparse. Thus, it might be expected that the region would have a greater uncertainty. It should be noted that the uncertainty quantification of IDT in HRRST and a feasible independent temperature measurement in the future are expected to further improve the correlation overall. For instance, it is anticipated that a better independent temperature measurement would improve the lean iso-octane mixture correlations, and all the lower temperature richer cases of $n$-heptane. It is possible that the lower temperature richer cases for mixtures of $n$-heptane might also include some low temperature chemistry effects and thus would require a staged inverse L-W method, the subject of future refinement.

\section{SUMMARY AND CONCLUDING REMARKS}

In this study, the HRRST has been used to acquire high-temperature ignition delay data for iso-octane, $n$ heptane, and acetone in a wide range of pressure and equivalence ratios. While the naïve correlation based on constant temperature and pressure conditions in the HRRST shows disagreement with conventional shock tube data, the ignition delay times correlate well with published data when accounting for the state history in the shock tube using L-W correlation. An inverse $\mathrm{L}-\mathrm{W}$ algorithm is demonstrated to deconvolve the constant state ignition delay for these fuels using different initial guesses, based on the relaxed Newton iteration and the GA. The optimized solutions using various approaches seem to be very consistent with reference data, demonstrating the feasibility and usefulness of the method to deconvolve high-temperature ignition delay under constant states. It is expected with an independent temperature measurement available in the future, IDTs from more exotic fuels in a broader range of thermodynamic conditions, and accounting for effects of heat loss, etc. could be resolved; and the HRRST could provide a useful tool for low volume high throughput IDT testing and kinetic validation in high-temperature conditions.

We thank G. Wang for technical assistance. acknowledgment is made to the Donors of the American Chemical Society Petroleum Research Fund for partial support of this research. This material is based upon work partially supported by the U.S. Department of Energy's Office of Energy Efficiency and
Renewable Energy (EERE) under the Co-Optimization of Fuels \& Engines Program Award Number DE-EE0007985.

\section{BIBLIOGRAPHY}

1. Hanson, R. K.; Davidson, D. F. Prog Energy Combust Sci 2014, 44, 103-114.

2. Sung, C.-J., Curran, H. J. Prog Energy Combust Sci 2014, 44, 1-18.

3. Beerer, D.; McDonell, V.; Samuelsen, S.; Angello, L. J Eng Gas Turbines Power 2011, 133, 11502.

4. Dec, J. E. Proc Combust Inst 2009, 32, 2727-2742.

5. Tanaka, S.; Ayala, F.; Keck, J. C.; Heywood, J. B. Combust Flame 2003, 132, 219-239.

6. Lynch, P. T.; Wang, G. Proc Combust Inst 2017, 36, 307-314.

7. Livengood, J. C.; Wu, P. C. Symp Combust 1955, 5, 347-356.

8. Pan, J.; Zhao, P.; Law, C. K.; Wei, H. Int J Engine Res 2016, 17, 825-835.

9. Tao, M.; Han, D.; Zhao, P. Combust Flame 2017, 176, 400-408.

10. Yates, A.; Bell, A.; Swarts, A. Fuel 2010, 89, 8393.

11. Khaled, F.; Badra, J.; Farooq, A. In 10th US National Meeting on Combustion; College Park, MD, 2017; p. 3A10.

12. Szybist, J. P.; Wagnon, S. W.; Splitter, D.; Pitz, W. J.; Mehl, M. SAE Int J Engines 2017, 10, 24-61.

13. Tao, M.; Zhao, P.; DelVescovo, D.; Ge, H. Combust Flame 2018, 192, 238-249.

14. Miyoshi, A. Phys Chem Chem Phys 2018.

15. Reyes, M.; Tinaut, F. V., Andrés, C.; Pérez, A. Fuel 2012, 102, 289-298.

16. Lynch, P. T. Rev Sci Instrum 2016, 87, 56110.

17. Tranter, R. S.; Lynch, P. T. Rev Sci Instrum 2013, 84, 94102.

18. Burcat, A.; Ruscic, B. Gas Thermochemical Database with updates from Active Thermochemical Tables; 2015; [Internet]. http://garfield.chem.elte. hu/Burcat/burcat.html. [cited 2015 Jul 13].

19. Gardiner, W. C.; Walker, B. F.; Wakefield, C. B. In Shock Waves in Chemistry; Lifshitz, A., Ed.; Marcel Dekker: New York, 1981; p. 319.

20. Tang, W.; Brezinsky, K. Int J Chem Kinet 2006, 38, 75-97.

21. Mitchell, M. An Introduction to Genetic Algorithms; MIT Press: Cambridge, MA, 1999; pp. 62-75.

22. Jeffries, J. B.; Schulz, C.; Mattison, D. W.; Oehlschlaeger, M. A.; Bessler, W. G.; Lee, T.; Davidson, D. F.; Hanson, R. K. Proc Combust Inst 2005, 30, 15911599.

23. Laich, A.; Lynch, P. T. In 10th US National Meeting on Combustion; College Park, MD, 2017; p. 3A1.

24. Shen, H. P. S., Vanderover, J.; Oehlschlaeger, M. A. Combust Flame 2008, 155, 739-755. 
25. Oehlschlaeger, M. A.; Davidson, D. F.; Herbon, J. T.; Hanson, R. K. Int J Chem Kinet 2003, 36, 67-78.

26. Heyne, J. S.; Dryer, F. L. J Phys Chem A 2013, 117, 5401-5406.

27. Tranter, R. S.; Sivaramakrishnan, R.; Srinivasan, N.; Brezinsky, K. Int J Chem Kinet 2001, 33, 722-731.

28. Davidson, D. F.; Hanson, R. K. Int J Chem Kinet 2004, $36,510-523$.
29. Pichon, S.; Black, G.; Chaumeix, N.; Yahyaoui, M.; Simmie, J.; Curran, H.; Donohue, R. Combust Flame 2009, 156, 494-504.

30. Davidson, D. F.; Ranganath, S. C.; Lam, K.-Y., Liaw, M.; Hong, Z.; H, R. K. J Propuls Power 2010, 26, 280 287.

31. Chaos, M.; Dryer, F. L. Int J Chem Kinet 2010, 42, 143-150. 